\title{
Circulating tumor cells and their clinical significance
}

\author{
Ewa Anna Grzybowska, Anna Fabisiewicz
}

Metastases to other organs and the formation of secondary tumors are responsible for $90 \%$ of cancer-related deaths. However, even in the early stages of cancer, about 30-40\% of patients with localized disease may have latent metastasis, which are likely derived from circulating tumor cells (CTCs) involved in disease progression. Therefore, detection and analysis of CTCs can play an important role in the diagnosis and decision-making of adjuvant treatment that aims to prevent metastasis. At present, patients' selection of treatment is based on the statistical risk of recurrence of metastatic disease, without considering whether the tumor cells have spread from the primary tumor. This may lead to unnecessary treatment of non-metastatic disease patients. Therefore, early detection of CTCs in the blood is critically important, and should allow for a more accurate assessment of disease severity. Here, we provide an overview of CTC phenotypes, including plasticity of CTCs, and their clinical significance.

NOWOTWORY J Oncol 2017; 67, 4: 243-250

Key words: CTC, metastasis

\section{Introduction}

Circulating tumor cells (CTCs) have been detected in blood, and first described in 1896 by Thomas Ashworth [1]. CTCs are defined as circulating cells whose antigenic or genetic characteristics correspond to the characteristics of a specific tumor type. The presence of CTCs in the blood stream reflects the process of metastasis, as described by the theory of'seed and soil' [2]. Under this definition, metastasis occurs when cancer cells are detached from the primary tumor and enter the bloodstream, moving in the direction of distant organs where they can settle and create a new focus i.e., a metastasis [2].

Together, metastasis and the formation of secondary tumors are responsible for $90 \%$ of deaths in cancer patients. However, even in the early stages of cancer, about $30-40 \%$ of patients with localized disease may have latent metastases, potentially derived from CTCs involved in the progression of the disease $[3,4]$. Therefore, detecting and analyzing CTCs may play an important role in the diagnosis and decision to provide adjuvant therapy. Patients have been hitherto selected based on the statistical risk of metastatic relapse, without due consideration of whether cancer cells have spread from the primary tumor. This may result in unnecessary therapeutic treatment of patients without metastases and, consequently, serious side effects of such treatment. For this reason, early detection of CTCs is critically important, as this would allow for a more accurate assessment of the disease stage, and for monitoring effects of treatment. Compared to metastatic tissue biopsy, there are many advantages of isolating CTCs. For example, blood collection is easier, cheaper, less invasive for the patient, and can be repeated several times while tracking cancer treatment. A blood sample containing CTCs is often referred to as a 'liquid biopsy', and may serve as a proxy for metastatic cancer [5]. In the future, liquid biopsy could be introduced for diagnostic screening, thus allowing for cancer to be monitored more easily via blood testing.

\section{The biology of metastasis}

Metastatic colonization by CTCs is a complex process involving many steps, such as tissue invasion and intravasation, survival in the bloodstream, extravasation, organ infiltration, colonization of a metastatic niche, and formation of macrometastasis (Fig. 1).

Department of Molecular and Translational Oncology, Maria Skłodowska-Curie Memorial Cancer Center and Institute of Oncology, Warszawa, Poland 
A

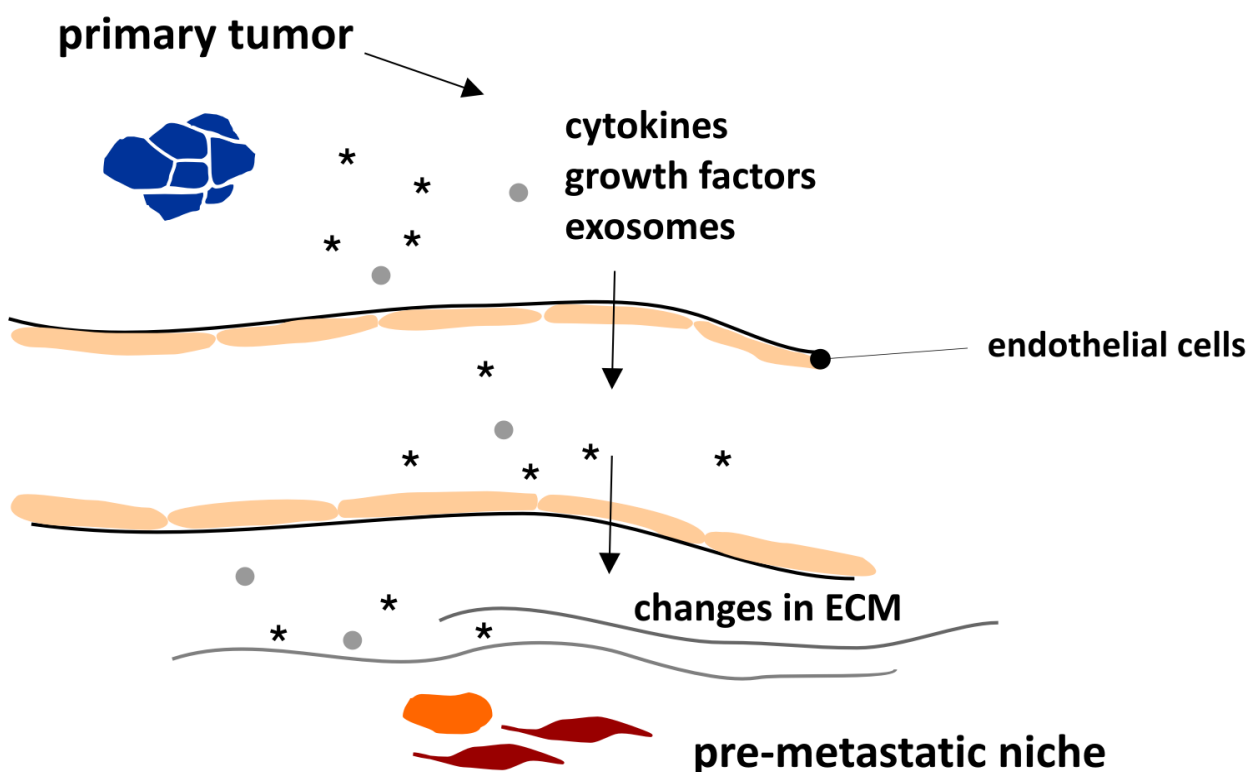

B

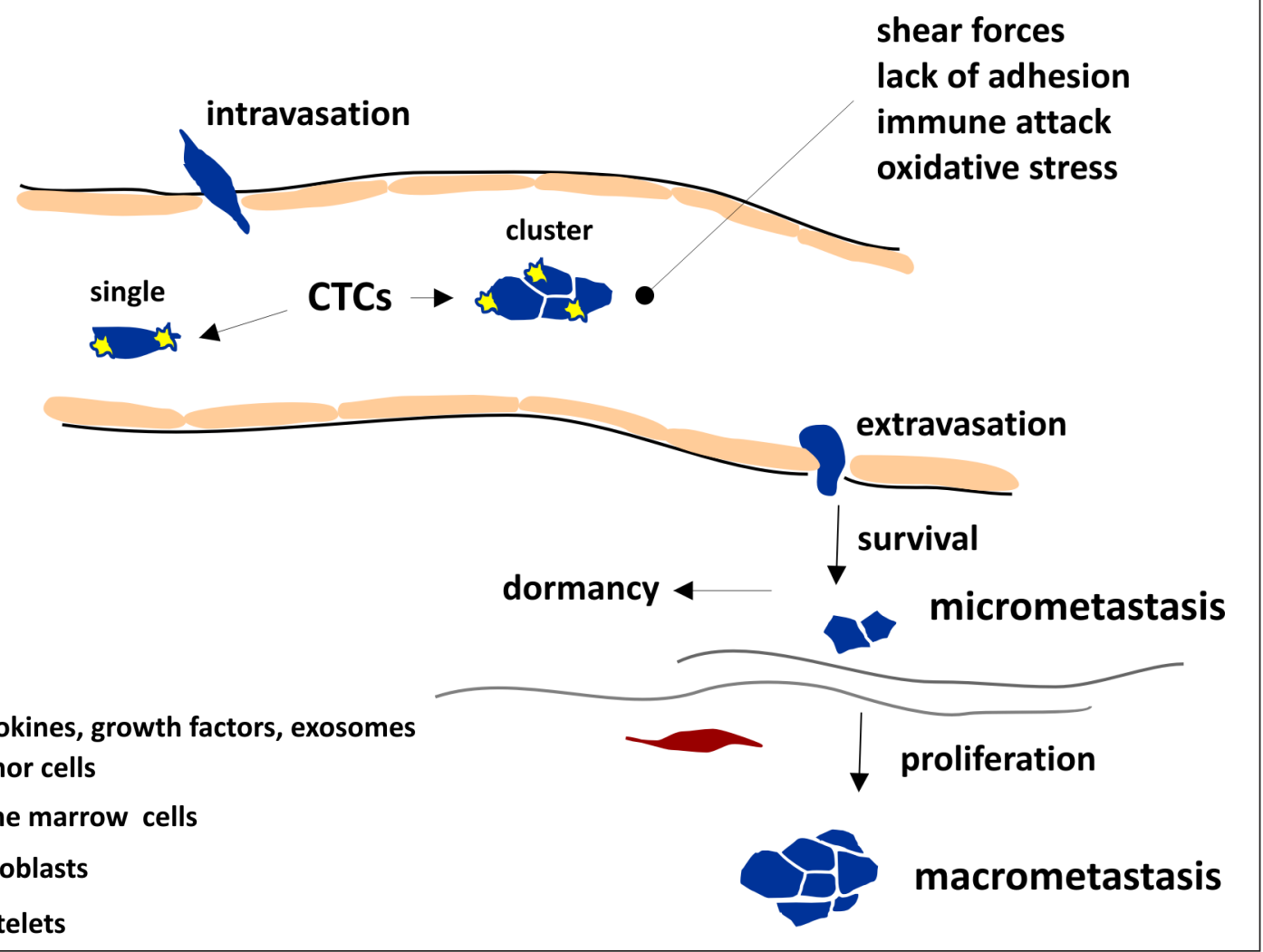

Figure 1. Metastatic cascade. A. Primary tumor secretes factors responsible for pre-metastatic niche formation at the distant site. This step facilitates subsequent tumor cell seeding by remodeling the composition of the extracellular matrix. B. Motile tumor cells (single cells and/or cell clusters) intravasate into circulation, where they become subjected to severe stress factors, including shear forces, lack of adhesion, immune reaction and oxidative stress. The influence of these factors can be modulated by platelets, which shield tumor cells from shear stress and immune attack. The lack of adhesion is compensated by either EMT or intracellular adhesion in case of CTC clusters. Subsequent extravasation into pre-metastatic niche allows for tumor cell survival and leads to either dormancy or proliferation and macrometastasis 


\section{Parallel progression}

Even small tumors at an early stage of advancement can shed cancer cells, which contradicts the conventional, linear model of cancer progression and dissemination [6]. In breast cancer, disseminated cancer cells (DCCs, also called DTCs, disseminated tumor cells) have been shown to contain fewer genetic alternations than primary cells, suggesting that they were seeded very early on and progressed separately. This phenomenon is known as the'theory of parallel progression', and poses important therapeutic problems, since cell dissemination occurs even before diagnosis [7]. Previous studies have shown that early DCCs are not as effective as late DCCs in the formation of primary tumors after implantation in the mammary fat pad. However, early DCCs are more metastasis-competent, and form more metastases and at a faster rate than late DCCs [6].

Early dissemination was demonstrated to depend on progesterone hormone signaling and abnormalities in the WNT signaling pathway [7]. As shown by Hosseini et al. [6], early DCCs have a different molecular signature than cells of the primary tumor. Further knowledge is needed to unveil the trigger mechanisms of early dissemination, which will be critical to future schemes of prevention and treatment.

\section{Routes of invasion}

For early or late cancer cells to disseminate, several vital problems need to be resolved; one of these problems invoIves efficient migration into the circulatory system.

The first step in the metastatic cascade is an invasion of surrounding tissues, which usually coincides with degradation of the stroma, activation of the immune system, and generation of cancer associated fibroblasts (CAFs) and the reactive stroma. These create a microenvironment that supports migration and cancer cell invasion. Cell migration can take many forms, such as the migration of single cells secreting extracellular-matrix (ECM) degrading enzymes, migration along collagen fibers [8] or nerve fibres [9], or collective migration in strands, sheets, or clusters. Most tumors are of epithelial origin, and epithelial cells are typically non-motile and do not secrete ECM-degrading enzymes, which is the hallmark of invasive cells. Thus, to acquire the ability to migrate and degrade ECM, cells can undergo an epithelial-mesenchymal transition (EMT). Epithelial cells that convert to a mesenchymal phenotype lose their apical-basal polarization, and thus their ability to adhere to neighbouring cells, acquire a spindle shape, and potential to secrete metalloproteinases [10]. EMT is triggered by transcription factors, such as SNAIL, SLUG, ZEB, KLF8, or Twist. During the process of EMT, the expression of proteins specific for epithelial cells (e.g., E-cadherin, cytokeratins and EpCAM) decreases, whereas expression of proteins characteristic for mesenchymal cells (e.g., vimentin and N-cadherin) increases. The ability to penetrate the basement membrane and extracellular matrix also increases. These are widely regarded as metastasis-promoting factors. However, the exact role of EMT in cancer dissemination is currently under debate. This is because, although EMT undeniably renders cells more motile and invasive, supports their survival in the bloodstream and protects them from anoikis (a form of apoptosis triggered by the lack of adhesion), several recent reports suggest that it is dispensable for metastasis - especially in breast and pancreatic cancers [11, 12]. It is also worth recalling that, to efficiently colonize a new metastatic site, cancer cells must reverse EMT and undergo the mesenchymal-epithelial transition (MET). This reversal further complicates the process.

Other than the mesenchymal and collective mode of migration used by cancer cells to spread, there is also ameboid cell migration, which is faster than the other types of migration and is adhesion-independent. Ameboid cells do not degrade ECM, but rather, squeeze through the ECM structures or cracks in cell layers. Ameboid migration also requires substantial phenotypic changes, usually in the form of a mesenchymal-ameboid transition (MAT).

In contrast to such modes of migration, collective movement of cancer cell clusters do not require extensive phenotype changes, since they retain the principal property of epithelial cells, which is the presence of strong cell-cell contacts. Presumably, this gives cancer cells an advantage in efficient seeding, given that they have been shown to have higher metastatic potential than single CTCs [13]. Indeed, polyclonal metastases have been documented in prostate cancer, and in mouse models of breast cancer [13, 14]. Although CTC clusters retain cell-cell contacts, studies have shown that they are not fully epithelial, but rather, display a hybrid epithelial-mesenchymal phenotype $[13,15]$. This hybrid phenotype may be crucial to their metastatic efficiency.

\section{CTC mobilization by treatment}

A comprehensive review by Martin at al. [16] summarizes the current knowledge on the effects of anti-cancer treatment on mobilization of CTCs into the circulation. It was shown that different types of treatment applied to cure locoregional disease (i.e., radiotherapy, surgery, systemic therapies) may actually contribute to the increase in the number of CTCs in patients' blood, or an increase in proliferative capacity of CTCs. One possible explanation of this finding is the detrimental effect of various anti-tumor therapies on tumor vasculature, which may facilitate the entry of cancer cells into the circulation. So far, no studies have proved that this effect impacts metastasis in patients, but certainly such an opportunity exists. Indeed, there are studies of metastasis in irradiated versus non-irradiated mice that point to this conclusion $[17,18]$. As addressed later in this review, such effects may interfere with assessing the prognostic powers of CTC count in patients, which should 
be taken into consideration. Further understanding of this topic may be critically important for deciding on treatment options, especially adjuvant therapies.

\section{In the circulation}

Hematogenous dissemination encompasses intravasation of cancer cells and their survival in the bloodstream. Intravasation is promoted by a number of factors, including crosstalk in secretion between tumor cells and perivascular macrophages, TGF- $\beta$ signaling, and the expression of Twist [19]. Following intravasation, cancer cells expose themselves to an extremely hostile environment and are exposed to many potentially damaging agents, such as shear forces, lack of adhesion, the innate immune response, and oxidative stress. As such, it is not surprising that survival of cancer cells in the bloodstream (or lymphatic system) is minimal. However, cancer cells have devised some forms of protection, including EMT and association with platelets, which shields them from both immune attack and hemodynamic shear forces. Platelets release TGF- $\beta$, inducing EMT [20], and they also secrete adenine nucleotides which relax endothelial cell junctions, which supports extravasation [21]. CTCs also undergo reversible metabolic changes, which helps them to withstand oxidative stress [22].

CTC flow is determined by patterns of blood circulation. This may help to explain why the lungs and liver are some of the most common target sites of metastasis, due to the venous circulation from most organs and the gut. Extravasation is thought to occur by mechanical entrapment of CTCS in capillaries and rupture of these vessels [23]. This mechanism could be especially effective for CTC clusters, when considering their size. CTC clusters were recently shown, however, to traverse capillary vessels, thereby reversibly re-organizing into a single-chain structure [24]. These data demonstrate that not all CTCs will end up being lodged within a capillary vessel. Rather, CTCs can extravasate by adhering to the vascular wall, and transmigrating to the other side of the endothelial cell layer.

\section{Seeding}

Infiltrating new tissue is the next and very inefficient step in cancer cell dissemination. Although CTCs extravastate and became DTCs, their chance of survival depends on the supportive metastatic niche in which they can proliferate, or at least survive and stay dormant. The creation of a pre-metastatic niche, even before the arrival of tumor cells, is promoted by factors secreted by the primary tumor (i.e., cytokines, growth factors, exosome components). These travel via the circulation to distant organs, and initiate structural and functional changes at a new site, which primarily encompass modifications to ECM components (i.e., fibronectin, tenascin C, collagens, galectins, periostin, osteopontin, versican, lysyl oxidase, MMPs) and the recruitment of bone marrow-derived cells [25]. Pre-metastatic changes create a permissive microenvironment, and allow some of the tumor cells to survive after extravasation. Nevertheless, a substantial proportion of tumor cells remain dormant. Further changes (e.g., immune, hormonal, or growth factor activation) may induce proliferation of these cells and produce macrometastasis.

\section{Clinical application}

To date, the only test approved by the FDA for measuring CTCs is the CellSearch system (Veridex, USA). Defined criteria for measuring CTCs and clinical study outcomes have led to the inclusion of the CellSearch test as a prognostic tool for breast cancer [26,27], colorectal cancer [28], and prostate cancer [29] in advanced stages of metastatic cancer. The number of preclinical and clinical studies on the possible applications of CTCs continues to grow, and studies have been conducted on both early stage disease and advanced cancer. These studies have focused primarily on using the CellSearch platform with RT-PCR. However, to date, no system based on CTCs has been approved to guide the appropriate choice of treatment and to monitor treatment efficacy. This may be because studies evaluating CTCs are often difficult to interpret, which may be due to various factors, including: different techniques for measuring CTCs, different criteria for defining CTCs (expression of various tumor markers), heterogeneity among enrolled patients, or a limited number of patients meeting required enrollment criteria. At present, the only FDA-approved clinical application of CellSearch is in estimating the chances of disease progression and/or survival. Indeed, the majority of research studies, including those reviewed by the FDA, confirm the prognostic value of CTCs for estimating clinical parameters, such as disease-free survival (DFS), overall survival (OS), and progression-free survival (PFS).

\section{CTCs as a prognostic factor: measurement of CTCs prior to treatment, and monitoring CTC levels after initiation of therapy}

Breast cancer

A landmark study by Cristofanilli et al. demonstrated that the amount of blood CTCs (measured by CellSearch) in women with metastatic breast cancer prior to the start of a new therapy differentiated patients at high vs low risk of rapid progression [26]. The cut-off point between the two groups was set at 5 CTC per $7.5 \mathrm{~mL}$ of peripheral blood. Other studies, with 75 patients have shown that this established cut-off point is also predictive of PFS and OS. Further studies have confirmed the prognostic value of CTCs, suggesting that it is possible to assess progression rates prior to undertaking antitumor therapy in patients with metastasis to distant organs [27, 30,31]. Taken together, these encouraging results provide the basis of further research for using CTCS in qualifying patients to more invasive therapies. 


\section{Prostate cancer}

A study on 231 patients with prostate cancer resistant to castration (CRPC) with distant metastases has shown the prognostic value of CTCs, according to OS rates [32]. This study contributed to the acceptance of CellSearch by the FDA for this group of patients [32]. In this study, elevated CTC levels (i.e. $\geq 5 \mathrm{CTCs} / 7.5 \mathrm{~mL}$ blood) measured by an assay prior to chemotherapy was associated with poorer OS than in patients who had CTC levels $<5$. This association has also been observed after the initiation of therapy. Survival was significantly longer among patients with CTC levels below the cut-off level, relative to patients with persistently high CTCs. For patients with CTC levels that increased in the follow-up to $\geq 5$ CTCs, OS was poorer compared to patients with persistently low levels ( $<5$ CTCs). This may be due to the resistance of the subpopulation of cancer cells to the applied treatment. Taken together, these results indicate that continual assessment of CTCs during therapy may allow for closer evaluation of treatment efficacy and/or a better survival profile than a single assessment of CTCs prior to treatment.

To confirm these assumptions, two clinical trials are currently underway through the Stand Up to Cancer and the Prostate Cancer Foundations [33]. These studies aim to evaluate the potential for changing levels of CTCs in the blood during therapy to predict disease course among patients with prostate cancer.

\section{Colorectal cancer}

One study used the CellSearch system to confirm distant metastases in 430 colorectal cancer patients undergoing chemotherapy [28]. Using an estimated cut-off point of $3 \mathrm{CTCs} / 7.5 \mathrm{~mL}$ blood, the authors found that CTCs showed prognostic value for both PFS and OS. As with the study on prostate cancer [32], a decrease in CTCs below the cut-off point after chemotherapy was associated with significantly longer PFS and OS, compared with rates observed in patients with persistently high CTCs. A study by Molnar et al. in colorectal cancer patients treated with chemotherapy reported a significantly higher number of single CTCs, doublets, and CTC clusters in treatment non-responders relative to responders [34]. Moreover, in the study by Molnar et al., higher levels of CTCs correlated with macroscopic progression [34].

\section{CTC as a substitute endpoint in clinical trials}

When planning clinical trials, endpoints are defined. Endpoints are variables that determine patient health status for those taking part in the trial, and may include: survival, death, quality of life (rating in scores), or myocardial infarction. Instead of endpoints, surrogate endpoints may be used. Surrogate endpoints may be indicators of physiological or pathological processes (e.g., blood pressure levels), and are also called 'biomarkers' [35]. CTCs as a potential surrogate endpoint may be beneficial may be useful for predicting disease progression in early and late stages of cancer, monitoring response to treatment, or minimizing molecular residual disease after therapy. Several clinical studies on the efficacy of anti-cancer therapy have evaluated CTCs as a potential surrogate endpoint. For example, one study found that measuring CTCs via the CellSearch system was more sensitive than radiological imaging as a prognostic factor among patients with advanced breast cancer [36]. Further, the authors found an association between high and persistent levels of CTCs and a shorter OS, whereas radiological scans showed no disease progression [36]. Based on these results, the authors concluded that CTCs may indeed be a superior surrogate endpoint measure, as they are highly reproducible and correlate more closely with OS than do changes determined via traditional radiology.

In another study, CTC levels reportedly predicted disease progression at 9-12 weeks after the initiation of therapy among patients with advanced breast cancer [37]. This technique is therefore more precise than FDG-PET and $\mathrm{CT}$, and predicted disease progression when radiographic imaging had little prognostic value for patients with CTC levels below the threshold level of 5/7.5 mL blood. Yet another study found that CTC levels measured early, prior to diagnostics (i.e., at 7-9 weeks) were strongly correlated with subsequent radiographic images [31]. Taken together, these results suggest that CTCs may be used together with radiographic imaging to evaluate response to therapy. However, for CTCs to be considered a reliable endpoint in clinical trials, further research is needed in this area. There is also a need for more accurate validation of the selected method for measuring these cells in a particular patient test group.

\section{CTCs for assessing effectiveness of the applied therapy}

Prognostic properties of CTCs measured by the CellSearch method have been used to further study how specific therapies may be selected. For example, therapies may be selected based on baseline levels of CTCs in the blood prior to therapy, or based on changes in CTC numbers during therapy.

These possibilities are currently being studied on a large scale in patients with metastatic breast cancer, through the SWOG S0500 trial (American South West Oncology Group) [38]. The purpose of the SWOG S0500 trial is to confirm whether persistently high CTC numbers ( $\geq 5 / 7.5 \mathrm{~mL}$ blood) after the first chemotherapy cycle are an indicator of disease progression and/or for a change in treatment strategy. The assumption is that unchanged blood levels of CTCs reflect cancer cell resistance to the adopted therapy. Therefore, patients with persistently high CTCs should shift to another treatment, whereas patients with changing levels 
should continue the current therapy. This strategy thus allows for assessment of whether early referral to alternative therapy improves OS and PFS. Unfortunately, however, preliminary phase III results are not as promising as expected. Although results have confirmed the prognostic value of CTCs after the first cycle of chemotherapy in predicting OS, they did not show clinical value for evaluating the effectiveness of therapy. Given that patients continued their therapy that was initiated prior to qualifying for the SWOG S0500 study, it was not possible to exclude the effect of therapy on CTCs in the blood. The effect of various therapies on CTCs has not yet been defined, and the available literature data are not consistent in this regard [31]. It is possible that after the first treatment cycle, it is too early to assess CTC fluctuations concerning treatment efficacy.

Another recently initiated study in this group of patients is the CirCe01 study, which is evaluating changes in CTCS (measured by CellSearch) as early resistance markers after the initiation of therapy [39]. Upon observing persistently high CTC levels after the third cycle of chemotherapy, some patients will be directed to an alternative treatment. After the first cycle of an alternative therapy, CTCs will again be monitored, and upon reaching $\geq 5 \mathrm{CTCs} / 7.5 \mathrm{~mL}$ of blood, patients will be redirected to yet another therapy. The premise of this study is to establish whether early detection of chemoresistance is possible by measuring CTCs in the blood. There is however, a risk that a too rapid interruption of targeted anti-cancer therapy will stimulate the biological activity of some cancer cell subpopulations, and that introducing alternative therapies will not yield the expected benefits.

Additionally, to establish an effective antineoplastic therapy, clonal evolution of cancer cells should be characterized by means of CTC phenotype features. Expression of tumor markers (e.g., ER, PG, HER2) may vary between primary tumor cells and distant metastases in breast cancer $[40,41]$. As a result, circulating blood cells with metastatic potential may provide a better genetic model for guiding appropriate therapy for a particular patient. The purpose of these studies is not only to identify patients who may benefit from a certain therapy, but also to discover the mechanisms responsible for cancer cells developing treatment resistance and identifying appropriate therapeutic targets. One such study is the DETECT III study, which includes patients with breast cancer without HER2 expression in the primary tumor, and at least one detected CTC cell expressing this receptor. The purpose of the DETECT III study is to determine the efficacy of lapatinib (an inhibitor of the tyrosine kinase receptors HER2 and EGFR) in a select group of patients [41].

Another strategy for choosing a therapy for advanced breast cancer is based on the CTC-Endocrine Therapy Index (CTC-ETI) test, which simultaneously measures CTCs in the blood and the expression of selected tumor markers (i.e.,
$\mathrm{ER}, \mathrm{BCL}-2, \mathrm{HER} 2, \mathrm{Ki}-67$ ) that are predictors of resistance and sensitivity to endocrine therapy [42]. The CTC-ETI test aims to identify patients with estrogen receptor (ER) expression, for whom endocrine therapy may be ineffective. Validating the CTC-ETI test could in the future be used as a criterion for choosing an appropriate therapy (i.e., endocrine vs chemotherapy) among patients with ER-positive breast cancer with distant metastases.

Several studies have found a correlation between the presence of CTCs and disease stage, among patients with primary colorectal cancer as well as patients with distant metastasis. In these studies, CTCs were measured via CellSearch after tumor removal but before chemotherapy, and the cut-off point was $\geq 2 \mathrm{CTCs} / 7.5 \mathrm{~mL}$ of blood. The proportion of patients with CTCs ranged from $5 \%$ to $25 \%$ at stages I-III, and $62 \%$ at distant metastasis stage $[43,44]$. One study showed no CTCs after surgery among patients at stages I-III using the same cut-off point [45]. An interesting correlation was found in a homogeneous but small group of 37 patients at stage II and III, such that CTC levels were higher in patients at high risk relative to lower risk patients of the same stage [46]. These results highlight the potential use of CTCs among patients with metastatic colorectal cancer. Specifically, CTCs may be useful for guiding the application of more or less aggressive therapy for stage III, and introducing adjuvant chemotherapy for high risk stage II patients.

\section{CTCs in localized tumors without distant metastases}

Thus far, the study outcomes when using CTCs in patients with stage I-III breast cancer have not been clear in terms of the number of detectable CTCs. However, these studies do suggest that measuring CTCs before and during therapy may be an additional factor predicting risk of local disease relapse, and can be measured in addition to more commonly assessed biomarkers (i.e., tumor size, lymph node involvement, HER2 amplification). Consequently, CTCs may be of clinical relevance in the early detection of residual disease. Consistent with this notion, one study demonstrated that CTCs with CK19 expression (as detected by RT-PCR measured mRNA levels) are an independent predictor of PFS and OS among patients with both involved and clear lymph nodes [47]. These results were subsequently confirmed in an independent study of 167 patients with uninvolved lymph nodes undergoing chemotherapy [48]. CTCs were monitored in $23 \mathrm{~mL}$ of peripheral blood in the German SUCCESS study, which includes 1,500 patients with metastatic and non-metastatic breast cancer at high risk and treated with chemotherapy. In that study, they found a correlation between the presence of $\geq 1 \mathrm{CTC} / 23 \mathrm{~mL}$ of blood before chemotherapy and the number of lymph nodes involved, and detection of $\geq 1$ CTC during chemotherapy subsequently predicted DFS and OS [32]. In another study of 404 patients 
undergoing tumor resection, the presence of CTCs prior to surgical intervention was associated with a slightly higher likelihood of local recurrence (78.9\% for a 4 -year period without recurrence) compared to patients without CTCS (88.4\%) [49]. However, the small difference in outcomes between groups and the occurrence of metastases among patients without CTCs do raise some concerns about whether the CellSearch method can be used for determining the risk of recurrence for this group of patients.

\section{Clinical application of CTCs: Summary}

The lack of prospective studies on a homogenous and large group of patients together with the lack of knowledge about the effects of different therapies on CTC rates, preclude us from assessing whether a clinical application of CTCs is possible. When examining these applications among breast cancer patients, it appears to be particularly important that patients are adequately stratified according to their cancer subtypes. In 2017, the first European statistical analysis of the potential predictive value by CellSearch was published [50]. The analysis included 1,944 patients with metastatic breast cancer, from 51 centers. Using the cut-off threshold of 5 CTCs/7.5 mL blood, results clearly indicated that detection of CTCs - both before and during treatment — was associated with worse prognosis. Furthermore, CTCs measured prior to any new treatment constitute independent prognostic markers that could supplement the currently used clinical predictive biomarkers of breast cancer. It is also noteworthy that predicted disease progression was more accurate when based on measurement of CTC levels after the initiation of therapy, compared to predictions based on levels of tumor markers recommended for monitoring metastatic breast cancer according the international guidelines (e.g., CEA, CA15-3) [51]. This is the first analysis carried out in a large group of patients in Europe, and demonstrates the utility of CTCs in monitoring the progression of distant metastatic breast cancer, and accurately determining the risk of disease progression in a given patient.

\section{Perspective}

CTCs are expected to be routinely used in the next ten years alongside other clinical methods and markers (i.e., imaging techniques, serum markers) for tracking tumor growth over time [52]. We expect that fluid biopsy outcomes from a patient's blood obtained via modern and automated methods will enable the clinician to detect the cancer, determine its severity, and establish therapeutic goals. These outcomes will also allow for more personalized and modifiable treatments, based on the qualitative and quantitative monitoring of CTCs [53]. Moreover, more in-depth molecular research on CTCs broadens the knowledge-base on the biology of metastasis, which also has important implications for treating cancer patients.

\section{Abbreviations}

CAFs - cancer associated fibroblasts

CEA - carcinoembryonic antigen

$\mathrm{CK}$ - cytokeratins

CRPC - cancer resistant to castration

CTC - circulating tumor cells

DCCs - disseminated cancer cells

DTCs - disseminated tumor cells

ECM - extracellular-matrix

EMT - epithelial-mesenchymal transition

EpCAM - epithelial adhesion molecule

EpCAM - epithelial cell adhesion molecule

FDA — Food and Drug Administration

$\mathrm{FU}$ - follow up

HER2 - human epidermal growth factor

IF — immunofluorescence,

MAT - mesenchymal-ameboid transition

$\mathrm{MBC}$ - metastatic breast cancer

mCRPC - metastatic castration-resistant prostate cancer

MET - mesenchymal-epithelial transition

OS - overall survival

PFS - progression-free survival

RT-PCR - reverse transcription-PCR

TGF- $\beta$ - transforming growth factor beta

\section{Acknowledgments}

This work was financed by the National Science Centre [grant 2016/21/B/NZ2/03473].

Conflict of interest: none declared

\section{Anna Fabisiewicz, PhD}

Department of Molecular and Translational Oncology

Maria Sklodowska-Curie Memorial Cancer Center

and Institute of Oncology

Roentgena St. 5

Warszawa 02-781, Poland

e-mail:anna.fabisiewicz@coi.pl

Received: 21 Jun 2017

Accepted: 31 Sept 2017

\section{References}

1. Ashworth T. A case of cancer in which cells similar to those in the tumours were seen in the blood after death. Aust Med J 1869; 14: 146-149.

2. Paget $\mathrm{G}$. Remarks on a case of alternate partial anaesthesia. Br Med J 1889; 1: 1-3.

3. Braun S, Pantel K, Müller P et al. Cytokeratin-positive cells in the bone marrow and survival of patients with stage I, II, or III breast cancer. N Engl J Med 2000; 342: 525-533.

4. Clare SE, Sener SF, Wilkens W et al. Prognostic significance of occult lymph node metastases in node-negative breast cancer. Ann Surg Oncol 1997; 4: 447-451.

5. Alix-Panabières $C$, Vendrell JP, Slijper $M$ et al. Full-length cytokeratin-19 is released by human tumor cells: a potential role in metastatic progression of breast cancer. Breast Cancer Res 2009; 11: R39-R39.

6. Hosseini H, Obradovic MM, Hoffmann M et al. Early dissemination seeds metastasis in breast cancer. Nature 2016.doi:10.1038/nature20785.

7. Ghajar CM, Bissell MJ. Metastasis: pathways of parallel progression. Nature 2016. doi:10.1038/nature21104. 
8. Roh-Johnson M, Bravo-Cordero JJ, Patsialou A et al. Macrophage contact induces RhoA GTPase signaling to trigger tumor cell intravasation. Oncogene 2014; 33: 4203-4212.

9. Magnon C, Hall SJ, Lin J et al. Autonomic nerve development contributes to prostate cancer progression. Science 2013; 341: 1236361.

10. Vincent-Salomon A, Thiery JP. Host microenvironment in breast cancer development: epithelial-mesenchymal transition in breast cancer development. Breast Cancer Res 2003; 5: 101-106.

11. Fischer KR, Durrans A, Lee $S$ et al. Epithelial-to-mesenchymal transition is not required for lung metastasis but contributes to chemoresistance. Nature 2015; 527: 472-476.

12. Zheng X, Carstens JL, Kim J et al. Epithelial-to-mesenchymal transition is dispensable for metastasis but induces chemoresistance in pancreatic cancer. Nature 2015; 527: 525-530.

13. Aceto N, Bardia A, Miyamoto DT et al. Circulating tumor cell clusters are oligoclonal precursors of breast cancer metastasis. Cell 2014; 158 : 1110-1122.

14. Cheung KJ, Padmanaban V, Silvestri V et al. Polyclonal breast cancer metastases arise from collective dissemination of keratin 14-expressing tumor cell clusters. Proc Natl Acad Sci USA 2016; 113: E854-863.

15. Jolly MK, Boareto M, Huang B et al. Implications of the hybrid epithelial/mesenchymal phenotype in metastasis. Front Oncol 2015; 5: 155.

16. Martin OA, Anderson RL, Narayan K et al. Does the mobilization of circulating tumour cells during cancer therapy cause metastasis? Nat Rev Clin Oncol 2017; 14: 32-44.

17. Sheldon PW, Fowler JF. The effect of low-dose pre-operative X-irradiation of implanted mouse mammary carcinomas on local recurrence and metastasis. Br J Cancer 1976; 34: 401-407.

18. Camphausen K, Moses MA, Beecken WD et al. Radiation therapy to a primary tumor accelerates metastatic growth in mice. Cancer Res 2001; 61: 2207-2211.

19. Yang J, Mani SA, Donaher JL et al. Twist, a master regulator of morphogenesis, plays an essential role in tumor metastasis. Cell 2004; 117: 927-939.

20. Labelle M, Begum S, Hynes RO. Direct signaling between platelets and cancer cells induces an epithelial-mesenchymal-like transition and promotes metastasis. Cancer Cell 2011; 20: 576-590.

21. Schumacher D, Strilic B, Sivaraj KK et al. Platelet-derived nucleotides promote tumor-cell transendothelial migration and metastasis via P2Y2 receptor. Cancer Cell 2013; 24: 130-137.

22. Piskounova $E$, Agathocleous $M$, Murphy $M M$ et al. Oxidative stress inhibits distant metastasis by human melanoma cells. Nature 2015; 527: 186-191.

23. Al-Mehdi AB, Tozawa K, Fisher AB et al. Intravascular origin of metastasis from the proliferation of endothelium-attached tumor cells: a new model for metastasis. Nat Med 2000; 6: 100-102.

24. Au SH, Storey BD, Moore JC et al. Clusters of circulating tumor cells traverse capillary-sized vessels. Proc Natl Acad Sci USA 2016; 113: 4947-4952.

25. Hoye AM, Erler JT. Structural ECM components in the premetastatic and metastatic niche. Am J Physiol Cell Physiol 2016; 310: C955-967.

26. Cristofanilli M, Budd GT, Ellis MJ et al. Circulating tumor cells, disease progression, and survival in metastatic breast cancer. $N$ Engl J Med 2004; 351: 781-791.

27. Cristofanilli M, Hayes DF, Budd GT et al. Circulating tumor cells: a novel prognostic factor for newly diagnosed metastatic breast cancer. J Clin Oncol 2005; 23: 1420-1430.

28. Cohen SJ, Punt CJ, lannotti N et al. Relationship of circulating tumor cells to tumor response, progression-free survival, and overall survival in patients with metastatic colorectal cancer. J Clin Oncol 2008; 26: 3213-3221.

29. Danila DC, Heller G, Gignac GA et al. Circulating tumor cell number and prognosis in progressive castration-resistant prostate cancer. Clin Cancer Res 2007; 13: 7053-7058.

30. Paoletti C, LiY, Muniz MC et al. Significance of circulating tumor cells in metastatic triple-negative breast cancer patients within a randomized, phase II trial: TBCRC 019. Clin Cancer Res 2015; 21: 2771-2779.
31. Liu MC, Shields PG, Warren RD et al. Circulating tumor cells: a useful predictor of treatment efficacy in metastatic breast cancer. J Clin Oncol 2009; 27: 5153-5159.

32. de Bono JS, Scher HI, Montgomery RB et al. Circulating tumor cells predict survival benefit from treatment in metastatic castration-resistant prostate cancer. Clin Cancer Res 2008; 14: 6302-6309.

33. FriedlanderTW, Premasekharan G, Paris PL. Looking back, to the future of circulating tumor cells. Pharmacol Ther 2014; 142: 271-280.

34. Molnar B, Floro L, Sipos F et al. Elevation in peripheral blood circulating tumor cell number correlates with macroscopic progression in UICC stage IV colorectal cancer patients. Dis Markers 2008; 24: 141-150.

35. Aronson JK. Biomarkers and surrogate endpoints. Br J Clin Pharmacol 2005; 59: 491-494.

36. Budd GT, Cristofanilli M, Ellis MJ et al. Circulating tumor cells versus imaging-predicting overall survival in metastatic breast cancer. Clin Cancer Res 2006; 12: 6403-6409.

37. De Giorgi U, Valero V, Rohren E et al. Circulating tumor cells and bone metastases as detected by FDG-PET/CT in patients with metastatic breast cancer. Ann Oncol 2010; 21: 33-39.

38. Smerage JB, Barlow WE, Hortobagyi GN et al. Circulating tumor cells and response to chemotherapy in metastatic breast cancer: SWOG S0500. J Clin Oncol 2014; 32: 3483-3489.

39. Helissey C, Berger F, Cottu P et al. Circulating tumor cell thresholds and survival scores in advanced metastatic breast cancer: the observational step of the CirCe01 phase III trial. Cancer Lett 2015; 360: 213-218.

40. Meng S, Tripathy $D$, Shete $S$ et al. HER-2 gene amplification can be acquired as breast cancer progresses. Proc Natl Acad Sci USA 2004; 101: 9393-9398.

41. Fehm T, Muller V, Aktas B et al. HER2 status of circulating tumor cells in patients with metastatic breast cancer: a prospective, multicenter trial. Breast Cancer Res Treat 2010; 124: 403-412.

42. Paoletti C, Muniz MC, Thomas DG et al. Development of circulating tumor cell-endocrine therapy index in patients with hormone receptor-positive breast cancer. Clin Cancer Res 2015; 21: 2487-2498.

43. Sastre J, Maestro ML, Puente J et al. Circulating tumor cells in colorectal cancer: correlation with clinical and pathological variables. Ann Oncol 2008; 19: 935-938.

44. Maestro LM, Sastre J, Rafael SB et al. Circulating tumor cells in solid tumor in metastatic and localized stages. Anticancer Res 2009; 29: 4839-4843.

45. Thorsteinsson M, Soletormos G, Jess P. Low number of detectable circulating tumor cells in non-metastatic colon cancer. Anticancer Res 2011; 31: 613-617.

46. Gazzaniga P, Gianni W, Raimondi C et al. Circulating tumor cells in high-risk nonmetastatic colorectal cancer. Tumour Biol 2013; 34: 2507-2509.

47. Stathopoulou A, Vlachonikolis I, Mavroudis D et al. Molecular detection of cytokeratin-19-positive cells in the peripheral blood of patients with operable breast cancer: evaluation of their prognostic significance. J Clin Oncol 2002; 20: 3404-3412.

48. Xenidis N, Perraki M, Kafousi $M$ et al. Predictive and prognostic value of peripheral blood cytokeratin-19 mRNA-positive cells detected by real-time polymerase chain reaction in node-negative breast cancer patients. J Clin Oncol 2006; 24: 3756-3762.

49. Franken B, de Groot MR, Mastboom WJ et al. Circulating tumor cells, disease recurrence and survival in newly diagnosed breast cancer. Breast Cancer Res 2012; 14: R133.

50. Bidard FC, Peeters DJ, Fehm T et al. Clinical validity of circulating tumour cells in patients with metastatic breast cancer: a pooled analysis of individual patient data. Lancet Oncol 2014; 15: 406-414.

51. Shiomi-Mouri Y, Kousaka J, Ando T et al. Clinical significance of circulating tumor cells (CTCs) with respect to optimal cut-off value and tumor markers in advanced/metastatic breast cancer. Breast Cancer 2016; 23: 120-127.

52. Diamandis EP, Pantel $\mathrm{K}$, Scher $\mathrm{HI}$ et al. Circulating cancer cells and their clinical applications. Clin Chem 2011; 57: 1478-1484.

53. Krebs MG, Hou JM, Ward TH et al. Circulating tumour cells: their utility in cancer management and predicting outcomes. Ther Adv Med Oncol 2010; 2: 351-365. 